\title{
Spacing judgments as an index of integration from context-induced relational processing: Implications for the free recall of ambiguous prose passages
}

\author{
LEONARD D. STERN, RICHARD G. DAHLGREN, and LINDA L. GAFFNEY \\ Eastern Washington University, Cheney, Washington
}

\begin{abstract}
The effect of information integration on the recall of ambiguous prose passages was investigated. In Experiment 1, subjects read ambiguous passages that were difficult to comprehend without titles. In judging the relative positions in the passages of pairs of test sentences, subjects performed better when they read passages headed by a suitable title than when they read untitled passages or received a title at the time of testing. In Experiment 2, subjects provided with a title at encoding also better discriminated complete old sentences from foils composed of fragments of two different old sentences than did subjects provided with no titles or with titles at the time of testing. These two tests index the degree of inter- and intrasentence information integration, respectively. Two findings indicated that integration affected free recall of an ambiguous passage. First, when the degree of integration of the passage's propositions was controlled, free recall of the passage was no different for subjects who did or did not know the passage's title at encoding. Second, inducing subjects to comprehend the passage's sentences individually, without relating them to one another, reduced free recall of the passage.
\end{abstract}

Presentation of a disambiguating title improves free recall of an ambiguous prose passage (Bransford \& Johnson, 1972; Dooling \& Lachman, 1971), but the underlying mechanism is not well understood (Alba, Alexander, Hasher, \& Caniglia, 1981; Einstein, McDaniel, Bowers, \& Stevens, 1984; Schwarz \& Flammer, 1981; Summers, Horton, \& Diehl, 1985). Evidence will be presented here that shows that a title promotes the integration of information from different sentences as well as from within each sentence of such passages and that this integration plays an important part in enhancing free recall of the passages. The evidence is interpreted as supporting a relational processing account of context effects on information encoding.

Various mechanisms have been proposed to account for a title's effect on memory of ambiguous passages, all of which stress encoding rather than retrieval processes. An encoding account seems appropriate because recall of ambiguous passages is enhanced when the title is introduced at the time of encoding rather than at the time memory is tested (Bransford \& Johnson, 1972; Dooling \& Mullet, 1973). According to one proposed mechanism, schematastructured sets of typical ideas or expectations abstracted

\footnotetext{
This research was supported in part by a grant from the Northwest Institute for Advanced Studies. The authors wish to thank Douglas Hintzman for his helpful comments on a previous version of this paper. They also are grateful to Nora Hannan for assisting with Experiment 1 and Shannon Foraker for assisting with Experiment 2. Requests for reprints should be addressed to the first author at the Department of Psychology, Eastern Washington University, Cheney, Washington, 99004.
}

from past experience-activated by the encoding context determine which information will and which will not be encoded, perhaps by directing a reader's attention to important aspects of a stimulus (R. C. Anderson \& Pichert, 1978; Neisser, 1967; Schwarz \& Flammer, 1981) or by providing a framework with empty slots for specific information (Abelson, 1981; Ausubel, 1963; Schank \& Abelson, 1977). This view implies that information relevant to an activated schema is more likely to be stored than is information unrelated to the schema (Bower, Black, \& Turner, 1979). Contradictory evidence comes from studies that have discovered information in memory unrelated to the schema active at encoding (Alba et al., 1981 ; R. C. Anderson \& Pichert, 1978; Hasher \& Griffin, 1978). In a study by Alba et al., for example, recognition of sentences from an ambiguous prose passage was no different for subjects who were and subjects who were not provided at the time of encoding with a title that improved the passage's comprehensibility. Alba et al. concluded that "a schema is not a necessary component of the encoding process"' (p. 289).

The levels-of-processing framework (Craik \& Tulving, 1975) offers an alternative interpretation. According to this view, context allows a deeper or more elaborate encoding of a passage's propositions (J. R. Anderson \& Reder, 1979; Bradshaw \& J. R. Anderson, 1982; Morris, Stein, \& Bransford, 1979). Such an account is reasonable because attending to and elaborating on a stimulus's meaning promotes memory of the stimulus (Craik \& Tulving, 1975), and because subjects who read ambiguous prose passages generally indicate that knowledge of the 
passage's title aids their comprehension of the passage (which presumably facilitates its semantic or elaborative encoding). The elaboration interpretation is weakened, however, by the lack of evidence that context clearly and consistently benefits recognition memory of ambiguous material (Alba et al., 1981; Birnbaum, Johnson, Hartley, \& Taylor, 1980; Bower, Karlin, \& Dueck, 1975). This contrasts with findings that semantic or elaborative processing can benefit recognition memory as well as free recall (Craik \& Tulving, 1975; Jacoby \& Craik, 1979; Jacoby \& Dallas, 1981).

The concept of semantic or elaborative processing has been refined by distinguishing between relational and item-specific processing (Einstein \& Hunt, 1980; Hunt \& Einstein, 1981). Relational processing, which establishes interconnections among the encoded items, is proposed to enhance primarily free-recall performance by facilitating generation at the time of retrieval. Item-specific processing, which elaborates on each individual item being processed, is proposed to establish distinctively encoded information and thus to enhance primarily recognition performance by allowing accurate discrimination of old and new items during testing. According to this account, title information will facilitate relational processing and, thus, free recall of propositions in an ambiguous passage by inducing their interpretation as a meaningful whole (Kintsch \& van Dijk, 1978). Recognition of these propositions will not be strongly affected if equivalent proposition-specific processing occurs as subjects read the passages either with or without a title or if, for the subjects receiving a title, there is the kind of tradeoff between relational and item-specific processing that Summers et al. (1985) propose.

Although this account is appealing, there is little direct evidence for the assumption that a title promotes integration of the propositions of an ambiguous passage through relational processing or that the integration resulting from relational processing facilitates free recall of the passage. In the research presented here, we seek to provide such evidence; that is, we attempt to demonstrate that a disambiguating title aids relational processing, and thus the integration of a passage's propositions in memory, and that the increased integration contributes to improved free recall of these propositions.

We use temporal judgments to detect relational processing. That is, we expect relational processing to facilitate accurate judgments of the order in which a passage's propositions were presented, a possibility supported by studies of temporal coding (Tzeng \& Cotton, 1980; Winograd \& Soloway, 1985). Winograd and Soloway, for example, found that when a subject related an event to a previous event at the time of encoding, judgment of the events' relative recencies improved. The same outcome should occur if context enhances a person's tendency to relate propositions from a passage. Context may be expected to enhance this sort of relational encoding by providing a cohesive framework for interpreting the passage's propositions. Thus, if one knows that a passage describes fly- ing a kite, a phrase such as "birds seldom get too close" could be related meaningfully to the previous phrase "a seashore is a better place than a street," so that an integrated representation is formed of a kite flying at a beach in the presence of birds. Even though the resulting integrated representation might not directly include information about the temporal order in which component ideas were encountered, in accord with a multiattribute view of memory (Underwood, 1983), some information about the events' temporal properties should be stored in memory. Temporal information may take the form of a reference to the earlier event in the mental representation of the subsequent event; for example, in the passage about a kite, the encoding of the phrase about birds might include some reference to the earlier occurrence of the phrase about the seashore. Assuming, then, that context promotes the extent to which propositions from a passage are relationally encoded, subjects given a suitable title for interpreting an ambiguous passage should subsequently judge the relative recencies of the passage's propositions more accurately than subjects not given this information.

To examine this hypothesis, subjects in Experiment 1 saw ambiguous passages either with or without a disambiguating title (the title and no-title conditions, respectively). They then took a sentence distance judgment (SDJ) test to assess their knowledge of the temporal ordering of the sentences from the passage. In this test, subjects were to indicate the approximate number of sentences that separated two sentences in the passage. If context helps to integrate information in memory by promoting relational encoding of its propositions, performance in the title condition should exceed that in the no-title condition. To ascertain whether any benefit derived from the title occurred at encoding rather than at retrieval, another group of subjects read the untitled passage and received the title just before performing the SDJ task (the delayed-title condition). If context had no effect at the time of retrieval, performance in the delayed-title condition should not differ from that in the no-title condition.

\section{EXPERIMENT 1}

In this study, subjects read one of two passages that were intended to be difficult to understand without a title because the passages lacked concrete referents. One passage was Bransford and Johnson's (1972) Making and Flying a Kite passage; the other was written for this study and described starting a car (see Appendix A for both passages). To help ensure that subjects in the three reading conditions (i.e., title, no-title, and delayed-title) processed their paragraphs in a similar manner, sentences were presented one at a time on a video monitor. Although we intended to have the reading strategies of the three groups be similar, some variation in reading time was permitted by allowing subjects to control the duration of each sentence's display. We expected the absence of a disambiguating title to require more processing time than the presence of the title. Each passage was presented twice 
to enhance memory of the passage and thus help avoid a floor effect in the difficult SDJ task.

\section{Method}

Subjects. Undergraduate students in upper division courses at Eastern Washington University served as subjects in the study. Participants received extra course credit as compensation. A total of 68 students were tested individually; 33 of them received the Making and Flying a Kite passage and 35 of them received the Starting a Car passage. The data of 3 subjects who received the car passage were discarded because they correctly guessed the title of the passage while reading it. For both passage conditions combined, this left 23 subjects in the title condition and 21 subjects each in the delayed-title and no-title conditions.

Materials. For each of the two passages, three versions of a test booklet were prepared. The first page of each contained instructions for reading a passage. The instructions for the title condition named the title of the story and stated that the title should be used to help understand the passage. The next page contained instructions for rating the passage's comprehensibility using a scale of 1 (the passage made very little sense) to 7 (the passage made a great deal of sense). For the no-title and delayed-title conditions, the next page of the booklets requested a brief description of the topic of the passage if one had occurred to the subject while the passage was being presented. For the title condition, this page of the booklet contained a filler task that involved judging the writing proficiency of the author of the passage. The next page of all booklets contained instructions for the SDJ task. The instructions for the delayed-title condition included the additional information about the title of the passage (i.e., that the passage had been about either making and flying a kite or starting a car). The next two pages of all booklets contained sentence pairs for the SDJ task. The kite passage contained 15 pairs of test sentences, of which 5 had been separated in the passage by no intervening sentences, 5 had been separated by one or two sentences, and 5 had been separated by three to five sentences. In constructing each test pair, care was taken to select sentences that did not provide obvious clues about whether or not one sentence had directly followed the other. In addition, to avoid allowing subjects to take advantage of primacy and recency effects, the first and last sentences from the passage were not included in any test pair. The sentence pairs were arranged in random order. The 12 sentence pairs for the passage about the car were constructed in a similar fashion: 4 were separated in the passage by no intervening sentences, 4 were separated by one or two sentences, and 4 were separated by three to five sentences.

Apparatus. An Apple II Plus computer equipped with an ApplePsych Software system ${ }^{1}$ was used to present the sentences. Stimuli were displayed on a 12 -in. NEC monitor. A response key labeled "yes" was interfaced with the computer, which was capable of timing responses to an accuracy of $1 \mathrm{msec}$. The subjects were seated approximately $50 \mathrm{~cm}$ from the monitor, and were free to adjust their distance from the screen and the position of the response key.
Procedure. The subjects were assigned randomly to a title, notitle, or delayed-title condition of one of the two passages. The subjects were given instructions for the passage presentation. These instructions informed them that they would be asked to read a short paragraph and then be asked some questions about it. It was explained that the dozen or so sentences in the passage would be presented one at a time on a video monitor and that they should press a response key as soon as they were ready to view the next sentence in the paragraph. They were told that they would see the paragraph twice. After reviewing the instructions and answering any questions, the experimenter started the computer program.

The experiment began with a display of the message "Ready for Sentences?" on the monitor. The subject then pressed the response key to begin the display of the first sentence. Thereafter, each time the subject pressed the response key, the sentence on display was replaced by the next sentence from the paragraph. When the last sentence in a passage had been displayed, a press of the response key caused the message "End of Paragraph" to appear. Following a 6-sec delay, the query "Ready to See Sentences Again?" appeared. The subject followed the same procedure to view the sentences a second time

After the passage had been viewed twice, the experimenter gave the subjects the comprehensibility rating form, followed by either the filler task (for the title condition) or the topic guessing task (for the no-title and delayed-title conditions). After completing these tasks, all subjects received instructions for the SDJ task. These instructions informed the subjects that they would see a number of sentence pairs taken from the passage they had read earlier and that they should decide how many other sentences had intervened in the passage between each of these pairs of test sentences. The subjects were instructed to check one of three boxes labeled to indicate that $0,1-2$, or 3-5 other sentences had intervened. The subjects completed this test at their own pace.

\section{Results and Discussion}

Mean reading times per sentence for Trials 1 and 2 for subjects in the three reading conditions (title, no-title, and delayed-title) and two passage conditions (kite and car) appear in Table 1 . These data were analyzed in a threeway analysis of variance (ANOVA) (trial $x$ passage $x$ reading condition), with trial as a within-subjects variable and passage and reading condition as betweensubjects variables. For all statistical analyses reported here, effects associated with an alpha level of .05 or less were considered significant.

To determine whether, regardless of passage and trial, receiving a title prior to reading the passages increased reading speed, mean per-sentence reading time for all subjects in the title condition was compared with that for subjects in the no-title and delayed-title conditions combined.

Table 1

Mean Reading Times (in Seconds) per Sentence by Reading Condition in Experiment 1

\begin{tabular}{|c|c|c|c|c|c|c|c|}
\hline \multirow[b]{3}{*}{ Trial } & \multirow[b]{3}{*}{ Passage } & \multicolumn{6}{|c|}{ Reading Condition } \\
\hline & & \multicolumn{2}{|c|}{ Title } & \multicolumn{2}{|c|}{ No-Title } & \multicolumn{2}{|c|}{ Delayed-Title } \\
\hline & & $M$ & $S D$ & $M$ & $S D$ & $M$ & $S D$ \\
\hline 1 & Kite & 4.79 & 1.92 & 5.17 & 1.94 & 5.09 & 2.46 \\
\hline 1 & Car & 4.46 & 1.83 & 6.46 & 4.52 & 6.44 & 2.22 \\
\hline 2 & Kite & 2.83 & 1.67 & 4.23 & 1.28 & 3.67 & 1.44 \\
\hline 2 & Car & 3.75 & 1.52 & 4.79 & 3.49 & 6.17 & 2.10 \\
\hline \multicolumn{8}{|l|}{ Overall } \\
\hline Combined & & 3.96 & & 5.14 & & 5.06 & \\
\hline
\end{tabular}


The analysis revealed significantly faster processing by subjects in the title condition $\left[F(1,59)=4.25, M S_{\mathrm{e}}=\right.$ 566.84]. Further analysis showed that the reading time for subjects in the no-title condition did not differ significantly from that for subjects in the delayed-title condition $(F<1)$. In addition, because the two-way interactions of each of these comparisons with the variables trial and passage were not significant (all $F_{\mathrm{s}}<1$ ), these effects did not differ significantly over Reading Trials 1 and 2 or over the kite and car passages. Analysis of the main effect of trial revealed significantly faster processing on Trial 2 than on Trial $1\left[F(1,59)=46.47, M S_{\mathrm{e}}=72.78\right]$. The interaction of the trial and passage variables was not significant $(F<1)$. In sum, these analyses reveal that titling these passages increased reading speed and that, in all reading conditions, processing was faster during the second than during the first presentation of each passage.

Mean comprehensibility ratings for passages in the title, no-title, and delayed-title conditions are shown in the first three rows of the body of Table 2 . These data were analyzed in a two-way ANOVA (passage $\times$ reading condition). In accord with the results of previous published studies, the subjects' ratings of their comprehension of the passages were significantly higher in the title than in the combined no-title and delayed-title conditions $[F(1,59)$ $=61.41, M S_{\mathrm{e}}=1.59 \mathrm{]}$. There was no significant interaction between this comparison involving the three reading conditions and the variable passage $(F<1)$. Thus, for both passages, knowledge of the title at the time of encoding improved comprehension. An additional analysis showed that the rated comprehension of subjects in the no-title and the delayed-title conditions was not significantly different $(F<1)$.

Of greatest importance to the hypothesis being investigated here was performance in the SDJ task. Performance in this task was assessed using two measures. One was the proportion of correct responses, that is, the proportion of sentence pairs that were correctly classified as having been separated by $0,1-2$, or 3-5 other sentences. The other measure was the correlation between the judged and actual number of sentences that had intervened between members of each test pair. To obtain this correlation, the values $0,1-2$, and 3-5 for the judged and actual separation of sentence pairs, were recoded to the values 1,2 , and 3 , respectively, and a value of $r_{s}$ was calculated for each subject, using the procedure described in Hays (1973, p. 791) to correct for ties. This measure (cf. Naveh-Benjamin, 1990; Tzeng, Lee, \& Wetzel, 1979) was expected to be a sensitive indicator of performance, since, unlike proportion correct, it takes into account the magnitude of a subject's error, in effect, penalizing a subject more, the more incorrect the response. Summary data for both measures of performance are shown in Table 2 . Statistical analysis of SDJ performance was conducted with a multivariate ANOVA with proportion correct and $r_{s}$ as the dependent variables and passage and reading condition as the independent variables. SDJ performance was better in the title than in the combined no-title and delayedtitle conditions [multivariate $F(2,58)=6.08$ ], but not significantly different for the no-title and delayed-title conditions [multivariate $F(2,58)=2.08, p>.10$ ]. This pattern of performance did not differ across the two passages (multivariate $F \mathrm{~s}<1.3$ ). These same outcomes were revealed in univariate ANOVAs conducted on the $r_{s}$ and proportion-correct measures individually. For $r_{s}$ and proportion correct, performance in the title condition exceeded that in the no-title and delayed-title conditions combined $\left[F(1,59)=5.95, M S_{\mathrm{e}}=.11\right.$, and $F(1,59)=12.24$, $M S_{\mathrm{e}}=.02$, respectively] and were not significantly different for the no-title and delayed-title conditions $\left(F_{\mathrm{s}}<1\right)$. Additionally, this pattern of performance for $r_{s}$ and proportion correct did not differ significantly for the two passages $(F s<1)$. Thus, the title improved performance in the SDJ task only when it was presented at the time of encoding. The enhanced ability to judge the relative positions of sentences in these previously read passages is seen as evidence that the context information aroused by the title helped to integrate these passages in readers' memories by promoting the relational encoding of each passage's component ideas.

Table 2

Mean Comprehensibility Ratings and SDJ Performance in Experiment 1

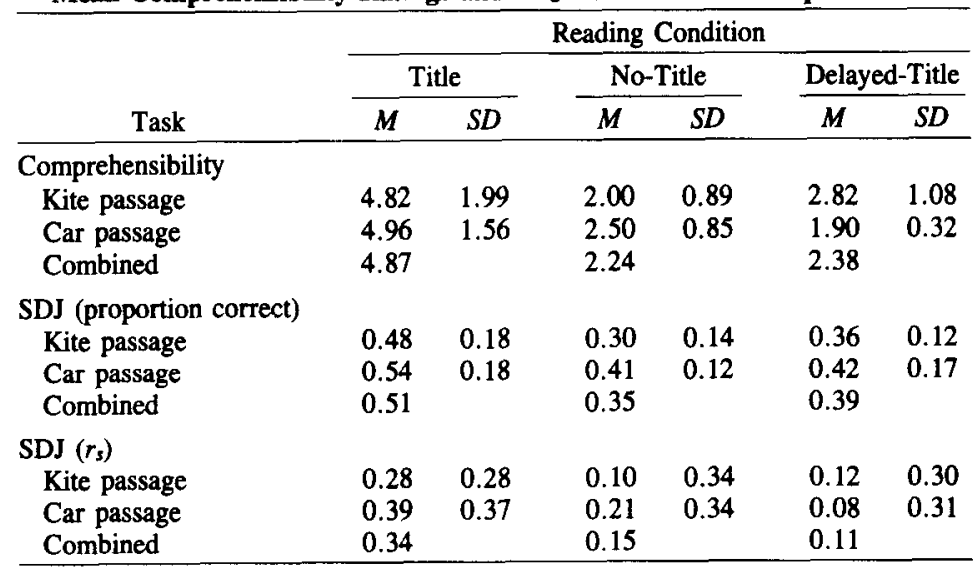




\section{EXPERIMENT 2}

Experiment 2 had two principal purposes. One was to determine whether a title, in addition to promoting integration in memory of information from different sentences of a passage, also facilitated integration in memory of information presented within individual sentences of a passage. To evaluate this, subjects were shown Bransford and Johnson's (1972) Washing Clothes passage (see Appendix B) and tested with a two-item forced-choice test having foils constructed from segments of two different sentences from the passage. For example, from the sentences First you arrange things into different groups depending on their makeup and Of course, one pile may be sufficient depending on how much there is to do, the foil was the sentence First you arrange things into different groups depending on how much there is to do. It was expected that if a title helped to integrate information presented within sentences, subjects in a title-reading condition would better discriminate old items in this composite foil (CF) task than would subjects in a no-title and delayedtitle reading condition.

The second purpose of Experiment 2 was to evaluate the relation between information integration and free recall. An important assumption made in Experiment 1 was that the integration in memory of a passage's propositions produced when a passage was relationally encoded is, to a large extent, responsible for aiding the passage's free recall. This assumed relation between free recall and association among to-be-recalled events often appears in models of memory (e.g., J. R. Anderson \& Bower, 1973; Raaijmakers \& Shiffrin, 1981). In our context, one implication of this relation is that when the degree of intraand intersentence information integration is equated statistically, the difference in free-recall performance of subjects who read a titled versus an untitled ambiguous passage should be reduced or eliminated. In Experiment 2 , this was tested by determining if the difference in free-recall memory of the Washing Clothes passage in the title and no-title reading conditions was reduced when $\mathrm{CF}$ and SDJ performance served as covariates in an analysis of covariance (ANCOVA).

Additional evidence for a causal relation between information integration and free recall was sought by presenting a different disambiguating title before each sentence in the Washing Clothes passage. This manipulation (the individual-sentence-title condition) was expected to make the sentences as understandable as in the title condition but to reduce the tendency of subjects to encode sentences relationally. The prediction was that both intersentence integration and free recall of subjects in the individual-sentence-title condition would be worse than that of subjects in the title condition. However, because subjects in the individual-sentence-title condition processed individual sentences meaningfully, their comprehension rating and their $C F$ performance was expected to be no different from those of subjects in the title condition. It should be noted that although subjects in the individual-sentence-title condition were expected to engage in relational processing of propositions presented within individual sentences and thus to develop associations among propositions from individual sentences, these intrasentence associations were expected to be of less fundamental importance to free recall of the Washing Clothes passage than were intersentence associations. The reason is that, given the structure of the Washing Clothes passage -12 sentences, each comprising an average of 1.5 propositions (see the subsequent Results and Discussion section)-longer chains of propositions from the passage could be encoded in memory inter- rather than intrasententially. On a more general level, the finding of equivalent memory performance of individual-sentence-title and title subjects in the CF task and different memory performance of these groups of subjects in the SDJ and freerecall tasks would indicate that manipulations of context information can affect different aspects of memory independently, and would reduce the possibility that enhanced SDJ performance in the title condition occurs because a title improves memory of the passage in general.

In addition to the title, no-title, and individual-sentencetitle reading conditions, Experiment 2 included the same delayed-title control reading condition used in Experiment 1 to help rule out the possibility that any memory performance advantage of subjects in the title condition was due to knowledge of the title at retrieval rather than at encoding.

Some minor changes in the procedure used for Experiment 1 were incorporated in this second study. To help ensure that none of the effects produced in the previous experiment were due to differences in sentence reading times among the title, no-title, and delayed-title conditions, sentences in Experiment 2 were presented at a fixed rate. In addition, to determine if the integrating effects of a title would become evident after a single reading trial, subjects in Experiment 2 were shown the passage only once.

\section{Method}

Subjects. One hundred eleven students at Eastern Washington University participated in this study for extra credit in a class. The subjects were tested in groups of 2 to 8 . The data of 7 subjects were discarded because they failed to follow instructions.

Materials. Each of the sentences of Bransford and Johnson's (1972) Washing Clothes passages was photographed and made into a slide. For the individual-sentence-title condition, a title was chosen that, in the judgments of the experimenters, was semantically ap propriate for the sentence and unrelated to the titles of all the other sentences (see Appendix B). For this individual-sentence-title condition, the slide showing each sentence had the title in a distinctive background and typeface centered two lines above the sentence.

As in the previous study, booklets were prepared for the different reading conditions. The booklets began with instructions for reading the passage, with the title included only in the instructions for the title condition. Subsequent pages in the booklets provided instructions and stimuli for the various memory tasks. For the freerecall task, the instructions given in the delayed-title condition mentioned that the passage had been intended to describe the process of washing clothes. The CF task was presented in the next two pages of all the booklets. Stimuli for this task consisted of five sentence 
pairs, each made up of an intact sentence from the passage and a foil composed of segments of two of the passage's other sentences. The foil was constructed so that, in the judgment of the experimenters, it was semantically appropriate for the passage. In each test pair, one of the two segments of the foil was identical to a segment of the intact sentence (see Appendix B). The foil in each test pair was assigned randomly as the first or second member, and each pair was followed by a 5-point confidence rating scale in which 1 was labeled as I'm just guessing and 5 was labeled as Certain I'm correct. The next page of all booklets presented instructions and test items for the SDJ task. The 12 sentence pairs used in this task were prepared in the same way as in Experiment 1. The last page of each booklet requested subjects to describe any strategy they might have used when reading the passage to help them remember it.

Procedure. The subjects were assigned randomly to one of the four reading conditions and tested in groups of up to 8 subjects, all of whom were in the same randomly determined reading condition. The experimenter began by giving instructions for viewing the sentences. For the title condition, these instructions mentioned that the passage described washing clothes and urged the subjects to try to use the title information to understand the sentences. The instructions for the no-title and delayed-title conditions just urged subjects to try to understand the sentences. For the individualsentence-title condition, the instructions mentioned that a title would accompany each sentence to help make it easier to understand. The subjects were not explicitly informed about the subsequent memory tests, only that they would later be asked some questions about the sentences. Subjects in all reading conditions were then shown the sentences one at a time at a fixed rate. The sentences were presented using a slide projector whose advance mechanism was controlled by an Apple IIE computer. For all reading conditions except the individual-sentence-title condition, the sentences were shown at a 7-sec rate; for the individual-sentence-title condition, a 9-sec rate was used, with the extra $2 \mathrm{sec}$ intended to allow for processing of the title that accompanied each sentence.

After viewing the sentences, the subjects were asked to indicate the number of sentences they did not have enough time to read and then to rate the comprehensibility of the sentences as a whole or, for the individual-sentence-title condition, to rate the comprehensibility of the individual sentences as a whole. Subjects in the notitle and delayed-title reading conditions were then asked to indicate what they had guessed the passage was about while reading it; subjects in the other two reading conditions were asked to perform the same filler task used in Experiment 1. Subjects in all reading conditions were then allowed $3 \mathrm{~min}$ to free-recall the sentences from the passage as accurately as possible. Approximately $3 \mathrm{~min}$ elapsed between presentation of the last sentence of the passage and the beginning of this free-recall task. The free-recall instructions for the delayed-title reading condition stated that the passage had been about washing clothes. The CF task was administered next, followed by the SDJ task, which the subjects completed at their own pace. This order of testing memory was kept the same for all subjects to prevent performance in a task from being aided by information from the passage presented as part of a previous test. After the subjects had completed the three memory tests, they were given the opportunity to describe any memory strategy they had consciously used while reading the sentences. Before being dismissed, the subjects were cautioned not to reveal the nature of the study to potential future participants.

\section{Results and Discussion}

No subject reported consciously applying what appeared to be a relational processing strategy when reading the sentences in anticipation of a memory test. Two subjects from the no-title reading condition correctly guessed the title of the passage, and their data were dropped from the study. Because the passage contained only two relatively long (23-word) sentences, any subject who had trouble reading more than two of the passage's sentences was considered an unreasonably slow reader, and their data were not included in subsequent analyses. This required removing the data of 2 subjects from the title-reading condition and the data of 3 from each of the other reading conditions. The mean number of sentences that the remaining subjects in the title, no-title, delayed-title, and individualsentence-title conditions reported having trouble reading adequately were $.51, .48, .29$, and .50 , respectively, values that did not differ significantly $(F<1)$. The number of subjects in the title, no-title, delayed-title, and individual-sentence-title reading conditions were 31,24 , 21 , and 24 , respectively.

The mean comprehensibility ratings given to the passage in the four reading conditions are shown in row 1 of Table 3. Statistical analyses showed that mean ratings given in the title condition were significantly higher than those given in the no-title and delayed-title conditions combined $\left[F(1,96)=9.47, M S_{c}=1.92\right]$, and that the mean ratings of the no-title and delayed-title subjects were not significantly different $(F<1)$. These expected results conform to the pattern found in Experiment 1. Further analysis showed that the mean comprehensibility rating in the individual-sentence-title condition did not differ significantly from that in the title condition $(F<1)$, which indicates that the individual titles made each sentence roughly as comprehensible as the Washing Clothes title did for the sentences as a whole.

Performance in the SDJ task was analyzed using proportion correct and the $r_{s}$ measure that was described in Ex-

Table 3

Mean Performance of Tasks in Experiment 2 as a Function of Reading Condition

\begin{tabular}{|c|c|c|c|c|c|c|c|c|c|}
\hline \multirow[b]{3}{*}{ Task } & \multirow[b]{3}{*}{ Measure } & \multicolumn{8}{|c|}{ Reading Condition } \\
\hline & & \multicolumn{2}{|c|}{ Title } & \multicolumn{2}{|c|}{ No-Title } & \multicolumn{2}{|c|}{ Delayed-Title } & \multicolumn{2}{|c|}{$\begin{array}{c}\text { Individual- } \\
\text { Sentence-Title }\end{array}$} \\
\hline & & $M$ & $S D$ & $M$ & $S D$ & $M$ & $\overline{S D}$ & $M$ & $S D$ \\
\hline Comprehensibility & & 5.35 & 1.43 & 4.27 & 1.45 & 4.38 & 1.15 & 5.15 & 1.44 \\
\hline \multirow[t]{2}{*}{ SDJ } & Proportion correct & 0.45 & 0.14 & 0.34 & 0.12 & 0.38 & 0.14 & 0.36 & 0.14 \\
\hline & $r_{5}$ & 0.29 & 0.35 & 0.15 & 0.26 & 0.11 & 0.38 & 0.14 & 0.38 \\
\hline \multirow[t]{3}{*}{$\mathrm{CF}$} & Proportion correct & 0.83 & 0.18 & 0.66 & 0.18 & 0.60 & 0.20 & 0.80 & 0.22 \\
\hline & Confidence correct & 4.25 & 0.80 & 4.23 & 0.78 & 3.91 & 0.83 & 4.26 & 0.56 \\
\hline & Confidence incorrect & 1.98 & 1.96 & 3.62 & 1.38 & 3.94 & 1.28 & 1.72 & 1.94 \\
\hline Recail & & 4.09 & 1.98 & 2.48 & 1.66 & 2.60 & 1.81 & 2.83 & 1.62 \\
\hline
\end{tabular}


periment 1 (see rows 2 and 3 of Table 3). As in Experiment 1 , with both measures as dependent variables, mean performance in the title condition was significantly higher than in the combined no-title and delayed-title conditions [multivariate $F(2,95)=4.12$ ]; performance in the no-title and delayed-title conditions did not differ significantly [multivariate $F<1.8$ ]. The same pattern of results held for each dependent measure of SDJ performance individually. Thus, these data are consistent with the view that a disambiguating title allows information from different sentences of a passage to become better integrated in memory. Furthermore, SDJ performance in the individualsentence-title condition did not differ significantly from that of the combined no-title and delayed-title conditions with $r_{s}$ and proportion-correct measures simultaneously serving as dependent variables (multivariate $F<1$ ) or with these measures analyzed separately (both $F \mathrm{~s}<1$ ).

Mean proportion-correct performance in the CF task for the four reading conditions are shown in row 4 of Table 3 . In accord with the hypothesis that a title enhances the integration in memory of intrasentence information, mean CF performance was significantly better in the title than in the no-title and delayed-title conditions combined $\left[F(1,96)=18.52, M S_{\mathrm{e}}=.95\right]$, but there was no significant difference between the no-title and delayed-title conditions $(F<1)$. In addition, mean proportion-correct performance in the title condition was not significantly different from performance in the individual-sentence-title condition $(F<1)$, indicating that the titles given to each individual sentence produced as much intrasentence information integration as the Washing Clothes title did for sentences read in the title condition. The same pattern of results appeared in subjects' mean confidence ratings of their performance in this task (see Table 3 , rows 5 and 6 ). Although there was no significant difference among the four reading conditions in confidence for correct responses $(F<1)$, for test sentences that were incorrectly selected, subjects in the combined-title and individualsentence-title conditions had significantly lower confidence than did subjects in the combined no-title and delayed-title conditions $\left[F(1,96)=18.14, M S_{\mathrm{e}}=2.90\right]$; within each of these combined reading conditions, the confidence ratings did not differ significantly $(F \mathrm{~s}<1)$. Thus, when a subject in the title or individual-sentencetitle condition incorrectly recognized a test sentence, the subject was more doubtful of the correctness of that selection than was a subject in the no-title or delayed-title condition. The increased doubt may reasonably be attributed to the memory representation of each sentence's being more fully integrated in the title and individualsentence-title conditions than in the no-title or delayedtitle condition.

The superior performance of subjects in the title condition of the CF task may possibly be due to their use of information about the distance between pairs of sentences from the passage. According to such a proposal, subjects could base decisions in the CF task on implicit judgments of the number of sentences that had separated the components of each test sentence in the pair, and select the sentence for which this implicit distance judgment provided the smaller value. ${ }^{2}$ Such a possibility seems unlikely, however, because there was no correlation between the combined indices of SDJ performance (proportion correct and $r_{s}$ ) and proportion-correct $C F$ performance for either the title condition $(R=.07)$ or all reading conditions combined $(R=.01$, both $F \mathrm{~s}<1)$; in addition, this mechanism receives no support from the finding that $C F$ performance was as good in the individual-sentence-title condition as in the title condition, whereas SDJ performance was as poor as in the no-title and delayed-title conditions. Thus, performance in the CF and SDJ tasks appears to reflect integration of different units of information from the passage.

One conclusion to be drawn from the finding of independence between our indices of intra- and intersentence relational processing within reading conditions is that subjects can exhibit enhanced relational processing of information from different sentences without exhibiting enhanced relational processing of information within each sentence. While this may seem surprising, such an outcome is possible if the smallest component of sentences is designated as a proposition, that is, a phrase that can be meaningfully interpreted. Because they can be understood in isolation, individual propositions in a multiproposition sentence may be related either across different sentences or within the same sentence. Thus, in the sentence from the Washing Clothes passage In the short run this may not seem important, but complications from doing too many can easily arise, the proposition about complications (i.e., complications from doing too many can easily arise) can be related to a proposition from a previous sentence of the passage about doing too few things at once, without first being related to the proposition about shortrun implications that occurred earlier in the same sentence. Given that we formed most of our foils in the CF task by combining semantically compatible propositions from different sentences, an insignificant positive relation between CF and SDJ performance within the individual reading conditions is conceivable.

Did integration of information from the passage affect its free recall? Free-recall performance was evaluated independently by two raters, who counted the number of "idea units" from the passage that each subject had reported. As in Bransford and Johnson's (1972) study, these idea units corresponded to sentences or phrases from the passage that expressed a coherent idea. A total of 18 idea units were identified in the passage, with no more than two of these appearing in a sentence. The correlation between the counts of the raters was .91; where there were disagreements between their counts, the mean of the two raters' values was used as the subject's recall score. The mean number of idea units recalled in each of the reading conditions is shown in Table 3 , row 7 . Statistical analysis showed higher recall in the title condition than in the other three reading conditions combined $[F(1,96)$ $\left.=14.16, M S_{e}=3.21\right]$ and no significant difference in 
mean recall among the other three reading conditions combined $(F<1)$.

To assess the degree to which integration of information from the passage affected its free-recall, mean freerecall performance of the title and no-title conditions was compared after controlling for the extent of intra- and intersentence information integration as indexed by proportion-correct CF performance, proportion-correct SDJ performance, and the $r_{s}$ measure of SDJ performance. These three covariates were each significantly correlated with free-recall performance of these two reading conditions combined (Table 4), and, in accord with the homogeneity of variance assumption of the ANCOVA, the test of heterogeneity of the regression hyperplanes (see, e.g., Huitema, 1980) was not significant $[F(3,47)$ $\left.=1.32, M S_{\mathrm{e}}=2.64, p>.10\right]$. The adjusted mean freerecall performance of the title and no-title reading conditions, taking the three indices of information integration into account, were 3.57 and 3.00 , respectively, values that did not differ significantly $\left[F(1,50)=1.20, M S_{\mathrm{e}}=2.69\right.$, $p>.10]$; before the adjustment, the mean free-recall scores of these two groups were 4.10 and 2.48 , respectively, values that differed significantly $[F(1,53)=10.28$, $\left.M S_{\mathrm{e}}=3.43\right]$. The index of intrasentence information integration (proportion-correct CF performance) and the combined indices of intersentence information integration (proportion-correct SDJ performance and the $r_{s}$ measure) each contributed significantly to adjusting the mean freerecall performance of these two reading conditions $[F(1,50)$ $=10.56, M S_{\mathrm{c}}=2.69$, and $F(2,51)=3.62, M S_{\mathrm{c}}=2.71$, respectively]. Thus, this analysis indicates that when intraand intersentence information is controlled, free-recall performance of title subjects becomes similar to that of the no-title subjects, an outcome that supports the assumption that information integration plays an important role in enhancing free recall of this passage.

Inclusion of the individual-sentence-title condition in this ANCOVA does not alter the outcome substantially: neither the heterogeneity of regression hyperplanes nor the recall differences among the three reading conditions is significant $\left[F(6,67)=.43, M S_{\mathrm{e}}=2.46, p>.10\right.$, and $F(2,73)=1.68, M S_{\mathrm{e}}=2.46, p>.10$, respectively]. However, inclusion of the delayed-title condition in the analysis introduces heterogeneity among the regression hyperplanes $\left[F(9,84)=2.49, M S_{\mathrm{e}}=2.55\right]$, a result produced by a nonsignificant negative correlation between free recall and the combined indices of information integration in just the delayed-title reading condition

Table 4

Intercorrelations Among the Three Covariates and the DV (Free-Recall Performance) Excluding the Effect of Reading Condition (Title, No-Title) in Experiment 2

\begin{tabular}{lccc}
\hline & $\begin{array}{c}\text { Proportion- } \\
\text { Correct CF }\end{array}$ & $\begin{array}{c}\text { Proportion- } \\
\text { Correct SDJ }\end{array}$ & $r_{s}$ SDJ \\
\hline Free recall & $.42^{*}$ & $.29 \dagger$ & $.30 \dagger$ \\
Proportion-correct CF & & .10 & .06 \\
Proportion-correct SDJ & & & $.73^{*}$ \\
\hline
\end{tabular}

$\left[F(3,17)=1.94, M S_{e}=2.87, p>.10\right]$. The absence of the positive correlation between indices of information integration and free recall found in the other reading conditions may reflect the use by delayed-title subjects of a strategy for performing the memory tasks that differed from that of subjects in other reading conditions. The delayed-title subjects may have relied heavily on the Washing Clothes title (provided to them after the passage had been encoded) while performing all the memory tasks, a strategy that was not equally effective in boosting both free recall of the passage and performance of the tasks measuring information integration.

Performance in the individual-sentence-title condition also suggested that integration contributed to the free recall of the passage. Presentation of individual titles for the sentences resulted in higher comprehensibility rating in the individual-sentence-title condition than in the combined no-title and delayed-title conditions $\left[F(1,96)=5.33, M S_{\mathrm{c}}\right.$ $=1.92$ ], but not for the title condition $(F<1)$. Analysis of performance in the CF task revealed that subjects in the individual-sentence-title condition were significantly more accurate than subjects in the no-title and delayed-title conditions combined $\left[F(1,96)=11.36, M S_{\mathrm{e}}=.95\right]$ but not significantly different from subjects in the title condition $(F<1.1)$. For the SDJ task, however, the combined proportion correct and $r_{s}$ measures revealed that performance was significantly less accurate in the individualsentence-title condition than in the title condition [multivariate $F(2,95)=3.72$ ], and not significantly different from that in the combined no-title and delayed-title conditions (multivariate $F<1$ ). These same outcomes were reflected in the univariate analyses of the individual measures of SDJ performance. For $r_{s}$ and proportion correct, performance in the individual-sentence-title condition was not significantly different from that in the no-title and delayed-title conditions combined (both $F \mathrm{~s}<1$ ) but differed significantly, or approached a significant difference, from performance in the title condition $[F(1,96)=$ 2.73, $M S_{\mathrm{e}}=.12, p<.10$, and $F(1,96)=7.09, M S_{\mathrm{e}}$ $=.02$, respectively].

These analyses of individual-sentence-title performance support the hypothesis that providing a different disambiguating title for each sentence of this passage induced subjects to interpret each sentence individually, and thus relationally process propositions presented within each sentence but not propositions presented in different sentences. In the title condition, however, because subjects were more likely to interpret individual propositions with respect to the passage as a whole, all propositions, both those presented in the same sentence and those presented in different sentences, were expected to be relationally processed. In accord with these expectations, performance of the CF task, a task intended to reflect intrasentence relational processing, was no different for subjects in the individual-sentence-title and title conditions. However, performance of the SDJ task, a task intended to reflect intersentence relational processing, was significantly better for subjects in the title condition than for those in the individual-sentence-title conditions. 
These outcomes are relevant to free recall because manipulation of the degree of relational processing affected free-recall performance: free-recall performance of the individual-sentence-title subjects was significantly worse than that of the title subjects $[F(1,96)=7.95$, $\left.M S_{\mathrm{e}}=3.21\right]$. Because free-recall performance of the individual-sentence-title subjects was no different from that of the no-title and delayed-title subjects combined $(F<1)$, it appears that intersentence relational processing was more critical than intrasentence relational processing to free recall of this passage, an outcome that is reasonable, because longer strings of propositions from a passage such as the one used here can be connected together through inter- rather than intrasentence associations. In spite of this relative importance of intersentence relational processing, it is surprising that mean free recall in the individual-sentence-title condition (2.83 idea units) was not significantly higher than that in the no-title condition (2.48), considering that, in the ANCOVA, CF performance contributed significantly to the adjustment of free-recall scores and CF performance was significantly better in the individual-sentence-title than in the no-title condition. One reason for this may be that the individual titles given at encoding reduced the tendency of subjects in the individual-sentence-title condition to engage in intersentence relational processing, so that intersentence associations were even less likely to be established in the individual-sentence-title than in the no-title condition. Compared with the no-title condition, this could have lowered free-recall performance in the individual-sentencetitle condition, thus offsetting free-recall gains resulting from their enhanced intrasentence relational processing. Detecting a difference between the level of intersentence relational processing of individual-sentence-title and notitle subjects is complicated by the possibility of floor effects in the measures of SDJ performance. For both the individual-sentence-title and no-title conditions, the proportion-correct measure indicates that performance in this task did not differ from chance (both $t \mathrm{~s}<1$ ), whereas by the $r_{s}$ measure, slightly more in keeping with a possible SDJ superiority of no-title over individualsentence-title subjects, mean performance of the no-title subjects differed significantly from zero $[t(23)=2.73]$, while that of individual-sentence-title subjects only approached a significant difference from zero $[t(23)=$ 1.86]. Of course, because the SDJ task was not performed by a control group that did not receive the passage, it is difficult to know whether a zero value for this $r_{s}$ measure represents chance performance. In any case, the absence of a free-recall advantage of individual-sentence-title over no-title subjects does not necessarily indicate that intrasentence associations were not important in the free-recall process.

\section{GENERAL DISCUSSION}

The results imply that the improved comprehension of prose passages derived from context information provided at the time the passages are encoded promotes the integra- tion in memory of information from the passages. One experimental test of this was based on the assumption that comprehension involves establishing meaningful relations among components of a stimulus passage (Kintsch, 1977) and on findings that memory for the presentation order of events is enhanced when the events are meaningfully related during encoding (Leonard \& Whitten, 1983; Tzeng \& Cotton, 1980; Winograd \& Soloway, 1985). Consistent with this assumption, we found that, compared with subjects who did not know the topic of the passage while they were reading it, subjects who knew the topic were subsequently better at judging the number of sentences that had intervened in the passage between pairs of test sentences (our SDJ task). We interpret this as evidence that context facilitated integration in memory of information from the passages. Additional evidence of the context's integrating influence came from the heightened ability of subjects to discriminate old, intact sentences from those composed of fragments of two old sentences (our CF task).

Two approaches were used to demonstrate that information integration affected free-recall performance. One involved statistically equating the inter- and intrasentence information-integration performance of subjects who read an ambiguous passage either knowing or not knowing the passage's title at encoding. The adjustment reduced the discrepancy in free-recall performance of these two groups of subjects, so they did not differ significantly. The other approach was to make the sentences of an ambiguous passage understandable to a group of subjects without encouraging them to engage in intersentence relational processing. This was achieved by preceding the presentation of each of the passage's sentences with a separate title unrelated to the titles of the other sentences. Although subjects in this individual-sentence-title condition indicated that they had comprehended the sentences no differently than subjects who had received a single title for the whole passage at encoding (the title condition), their freerecall performance was inferior to that of the title subjects and did not differ significantly from that of subjects who did not know the title at encoding. This outcome ties the free-recall enhancement to activities that involve intersentence relational processing.

In spite of these findings, performance on the SDJ and CF tasks might result from memory of the passage rather than from two forms of associations underlying free recall. That is, a title may improve memory of the passage in general, and this improvement is reflected in the performance of all tests of memory. Such a possibility seems to be contradicted by the disparity between the results of the CF task and free recall of Experiment 2. CF performance did not differ in the individual-sentence-title and title reading conditions, whereas free-recall performance did. A similar possibility is that performance in the SDJ task, rather than indexing intersentence associations, is derived from free-recall performance; that is, the heightened SDJ performance of subjects in the title conditions of our studies may result from their superior free recall of sentences. According to such an account, subjects ex- 
hibit enhanced SDJ performance because title information acts as an effective retrieval cue for sentences which, in turn, provide title subjects with a basis for computing distances between pairs of test sentences in the SDJ task. Even if one ignores the difficulty of explaining, without reference to intersentence relational processing and intersentence associations, how knowledge of individual sentences from the passage can be used to calculate distance between pairs of test sentences, this account is problematical. The SDJ and the preceding CF task, both recognition tasks, display sentences from the passage so that by the time a subject in Experiment 2 has seen half the SDJ test items, all sentences from the passage have been reintroduced to the subject (with many still visible on the test form). It seems unreasonable that if performance in the SDJ task depends on having sentences from the passage highly available in memory, subjects in the no-title and delayed-title conditions of Experiment 2 did not perform clearly above the chance level. Thus, we conclude that SDJ performance, rather than being based on free-recall performance, serves as an index of the intersentence associations that affect free recall.

The mechanism underlying inter- and intrasentence information integration probably stems from attempts to form cohesive mental structures during the comprehension process. According to this view, title or context information arouses general information in memory. This information facilitates attempts to form cohesive mental structures by providing potential relations among component elements of a stimulus passage (Kintsch, 1988; Kintsch \& van Dijk, 1978; Mandler \& Johnson, 1977; Smith, Adams, \& Schorr, 1978). This form of processing will enhance judgments of the relative recencies of sentences in a passage, since, as others have pointed out (e.g., Hintzman, Summers, \& Block, 1975; Tulving, 1983; Tzeng \& Cotton, 1980), when an event is related at the time of encoding to a previously encountered event, the temporal relationship between them can become better established in memory. Temporal information can be based on differences in the information associated with earlier and later events. Specifically, the original trace of the later event will include reference to the earlier event but the original trace of the earlier event will contain no such reference to the later event. Thus, title information for an ambiguous passage, by aiding the reader's ability to relate each currently encountered proposition to previous ones, can be expected to produce a more detailed specification in memory of the order in which the propositions were encountered.

Other mechanisms might underlie performance in a temporal judging task (Tzeng \& Cotton, 1980). For example, if one assumes that the general information aroused by title or context information takes the form of a schema and that schematic knowledge specifies the typical order of events (Minsky, 1975; Schank \& Abelson, 1977), then information about order of propositions in a text can possibly be derived from the structure of the schema. However, the temporal judgment performance of subjects in the delayed-title reading conditions of both our experiments indicates that this mechanism probably did not play a major role in our SDJ task. Subjects in this condition were given context information only at the time they judged the distances between pairs of test sentences. Because performance in this reading condition was no different from that of subjects not receiving any title information, it appears that arousal of relevant schematic information at the time of testing was not sufficient for enhancing performance in this task.

It also is possible to attribute the heightened performance on all the memory tasks exhibited by subjects in the title conditions of both our studies entirely to elaboration processes occurring during encoding, given that a number of researchers (e.g., J. R. Anderson \& Reder, 1979; Bradshaw \& J. R. Anderson, 1982; Morris et al., 1979; O'Brien \& Myers, 1985) have suggested that context facilitates remembering through elaborative processing. Such an account of our results, however, is unsatisfactory for a number of reasons. First, subjects in Experiment 2 who were given different disambiguating titles for each sentence of the passage (the individual-sentence-title condition) showed inferior free-recall and SDJ performance relative to that of subjects provided with a single title for the entire passage (the title condition). Because comprehensibility ratings given to the sentences in these two reading conditions did not differ, we assume that subjects in both reading conditions processed the sentences elaboratively. However, the free-recall and SDJ performance of subjects in the individual-sentence-title condition was inferior to that of subjects in the title condition and no different from those of subjects in the no-title and delayed-title conditions. Thus, elaboration alone does not seem to account for a title's effect on memory. Second, although elaboration seems capable of improving recognition of individual words and sentences (Fisher \& Craik, 1980; Jacoby \& Dallas, 1981; Schulman, 1974; Schustack \& J. R. Anderson, 1979; Stein, Littlefield, Bransford, \& Persampieri, 1984), Alba et al. (1981), using prose passages similar to the ones employed in our studies, found no evidence that knowledge of a title improved recognition of sentences from the passages. This makes an elaboration account of contexts' effects on memory less plausible. Finally, a more fundamental reason for questioning an elaboration interpretation of our data is the growing evidence that the nature of the associative processes underlying elaboration needs to be more clearly specified (Morris et al., 1979; Stein et al., 1984; Stein, Morris, \& Bransford,1978; Walker, 1986). For example, Stein et al. (1984) have shown that, contrary to earlier conceptualizations of the process of elaboration (Craik \& Tulving, 1975), increasing trace complexity with associatively related information does not necessarily improve retention. Einstein and Hunt (1980), in addressing the inadequacies of the concept of elaboration, have noted that it is useful to specify whether elaboration involves associating semantic or episodic knowledge with a to-be-remembered item. The terms item-specific and relational processing were in- 
troduced to underscore the importance of this distinction (Einstein \& Hunt, 1980), which has been used to explain various memory phenomena (Einstein et al., 1984; Hunt, Ausley, \& Schultz, 1986; Maki, 1990; McDaniel, Einstein, Dunay, \& Cobb, 1986; McDaniel, Einstein, \& Lollis, 1988; Summers et al., 1985).

The applicability of a relational processing account for the effect of a title on memory of ambiguous passages has been raised by others (e.g., Einstein et al., 1984; Summers et al., 1985). Our studies provide supporting evidence. We do not suggest, however, that a title's effect is necessarily limited to enhancing relational processing. Rather, we support Summers et al.'s view that title information is capable of affecting both relational and itemspecific processing. According to Summers et al., because subjects in a title condition generally do not expect a recognition test, they emphasize relational processing at the expense of item-specific processing, a tactic intended to facilitate free recall rather than recognition memory. Indeed, Summers et al. found that knowledge of an ambiguous passage's title improved sentence recognition of subjects who were led to expect a recognition test. Our data complement this view by providing more direct support for the notion that title information ordinarily enhances relational processing, resulting in a mental representation that is "assumed to be highly integrated and organized around the common features shared by the separate events"' (Hunt \& Einstein, 1981, p. 497).

Although Summers et al. present a reasonable justification that a title enhances item-specific processing, and thus recognition memory under certain circumstances, we think that their evidence must be interpreted with some caution. They tested recognition memory, in part, with "sentence-combination distractors," which were very much like our composite foils in Experiment 2. From our study, we concluded that the enhanced ability of subjects in the title reading condition to discriminate old sentences from composite foils was evidence of a heightened integration of old sentences in memory. Because Summers et al. mixed this kind of distrator with "word-substitution distractors," it is not clear that their recognition results reflect integration, which we view as a product of relational processing rather than enhanced item-specific processing.

It is worth noting that the temporal information underlying performance of the SDJ task is surface information, that is, information unrelated to a stimulus's meaning. Because some investigators (e.g., Bransford \& Franks, 1971; Peterson \& McIntyre, 1973) have concluded that increased comprehension and integration is associated with decreased memory for surface information, it may seem that performance in the SDJ task should be worse when title information increases a passage's comprehensibility. Evidence obtained by Gernsbacher (1985), however, indicates that improved comprehension can facilitate retention of surface information. Using pictures or words to present stories to subjects, Gernsbacher found better retention of picture orientation (Experiment 5) and word order (Experiment 6). Gernsbacher's account of these findings is similar in a number of ways to our account of our data. Gernsbacher suggests that in the comprehension process, a coherent mental structure is built up, in which newly encountered information that is related to previous information adds to the growing structure, keeping all information, including surface information, in an activated state, thus facilitating its subsequent accessibility. Gernsbacher's account, like ours, incorporates the notion that comprehension promotes the formation of integrated mental structures.

In sum, our data suggest that knowledge of the title of an abstract passage helps to integrate the passage in memory, and that this integration facilitates the passage's free recall. These findings support and clarify a number of other findings in the literature. Although our evidence is based on the use of passages that are difficult to understand because they lack concrete referents, it seems likely that context will have a similar integrating influence on other stimulus material. The techniques that we used to probe for integration-the sentence distance judgment and composite foil tasks-may be useful for detecting the integration of other stimuli.

\section{REFERENCES}

AbeLSON, R. P. (1981). Psychological status of the script concept American Psychologist, 36, 715-729.

Alba, J. W., Alexander, S. G, Hasher, L. Caniglia, K (1981). The role of context in the encoding of information. Journal of $E x$ perimental Psychology. Human Learning \& Memory, 7, 283-292

ANDERSON, J. R., \& BOWER, G H (1973). Human associative memory Washington, DC. Winston

ANDerson, J. R , Reder, L M. (1979) An elaborative processing explanation of depth of processing. In L. S Cermak \& F. I. M. Craik (Eds.), Levels of processing in human memory (pp. 385-403) New York Wiley

Anderson, R. C., \& Pichert, J W. (1978). Recall of previously unrecallable information following a shift in perspective. Joumal of Verbal Learning \& Verbal Behavior, 17, 12

AUSUBEL, D. P (1963). The psychology of meaningful verbal learning New York Grune \& Stratton.

Birnbaum, I. M., Johnson, M. K , Hartley, J. T \& \& TAYLor, T. H. (1980). Alcohol and elaborative schemas for sentences. Jourmal of Experimental Psychology: Human Learning \& Memory, 6, 293-300.

BOWER, G. H., BLACK, J B , \&URNER, T. J. (1979). Scripts in memory for text. Cognitive Psychology, 11, 177-220.

Bower, G. H , Karlin, M B., DueCK, A. (1975). Comprehension and memory for pictures. Memory \& Cognition, 3, 216-220.

Bradshaw, G L., ANDERSON, J R. (1982). Elaborative encoding as an explanation of levels of processing Journal of Verbal Learning \& Verbal Behavior, 21, 165-174

Bransford, J. D., Franks, J J. (1971). The abstraction of linguistic ideas. Cognitive Psychology, 2, 331-350.

Bransford, J. D., \& Johnson, M. K. (1972). Contextual prerequisites for understanding: Some investigations of comprehension and recall. Journal of Verbal Learning \& Verbal Behavior, 11, 717-726.

Craik, F. I. M., Tulving, E. (1975). Depth of processing and the retention of words in episodic memory Joumal of Verbal Learning \& Verbal Behavior, 11, 671-684.

DoOLING, D. J., \& LACHMAN, R. (1971). Effects of comprehension on retention of prose. Joumal of Experimental Psychology, 88, 216-222.

DoOLING, D. J , MULLET, R. L. (1973) Locus of thematic effects in retention of prose. Journal of Experimental Psychology, 97 , 404-406 
Einstein, G. O., \& HUNT, R. R. (1980). Levels of processing and organization: Additive effects of individual-item and relational processing. Joumal of Experimental Psychology: Human Leaming \& Memory, 6, 588-598.

Einstein, G. O., McDaniel, M. A., Bowers, C. A., \& Stevens, D. T. (1984). Memory for prose: The influence of relational and propositionspecific processing. Journal of Experimental Psychology: Leaming, Memory, \& Cognition, 10, 133-143.

Fisher, R. P., CRaIK, F. I. M. (1980). The effects of elaboration on recognition memory, Memory \& Cognition, 5, 400-404.

GerNSBACHER, M. A. (1985). Surface information loss in comprehension. Cognitive Psychology, 17, 324-363.

Hasher, L., * Grifin, M. (1978). Reconstructive and reproductive processes in memory. Journal of Experimental Psychology: Human Learning \& Memory, 4, 318-330.

Hays, Willuam L. (1973). Statistics for the social sciences. San Francisco: Holt, Rinehart \& Winston.

Hintzman, D. L., Summers, J. J., \& Block, R. A. (1975). Spacing judgments as an index of study-phase retrieval. Joumal of Experimental Psychology: Human Learning \& Memory, 1, 31-40.

HUITEMA, B. E. (1980). The analysis of covariance and alternatives. New York: Wiley.

Hunt, R. R., Ausley, J. A., \& Schultz, E. E. (1986). Shared and item-specific information in memory for event descriptions. Memory \& Cognition, 14, 49-51.

HUNT, R. R., EINSTEIN, G. O. (1981). Relational and item-specific information in memory. Journal of Verbal Learning \& Verbal Behavior, 20, 497-514.

JACOBY, L. L., CRAIK, F. I. M., (1979). Effects of elaboration of processing at encoding and retrieval: Trace distinctiveness and recovery of initial context. In L. S. Cermak \& F. I. M. Craik (Eds.), Levels of processing and human memory (pp. 1-22). Hillsdale, NJ: Erlbaum.

JACOBY, L. L., DALLAS, M. (1981). On the relationship between autobiographical memory and perceptual learning. Joumal of Experimental Psychology: General, 110, 306-340.

KINTsCH, W. (1977). On comprehending stories. In M. A. Just \& P. Carpenter (Eds.), Cognitive processes in comprehension (pp. 33-62). Hillsdale, NJ: Eribaum.

$\mathrm{KINTSCH}, \mathrm{W}$. (1988). The role of knowledge in discourse comprehension: A construction-integration model. Psychological Review, 95, 163-182.

KinTSCH, W., VAN DuK, T. A. (1978). Toward a model of discourse comprehension and production. Psychological Review, 85, 363-394.

LEONARD, J. M., \& WhitTEN, W. B. (1983). Information stored when expecting recall or recognition. Journal of Experimental Psychology: Learning, Memory, \& Cognition, 9, 440-455.

MAKI, R. H. (1990). Memory for script actions: Effects of relevance and detail expectancy. Memory \& Cognition, 18, 5-14.

MANDLeR, J. M., \& Johnson, N. S. (1977). Remembrances of things parsed: Story structure and recall. Cognitive Psychology, 9, 111-151.

McDaniel, M. A., Einstein, G. O., Dunay, P. K., \& Cobb, R. E. (1986). Encoding difficulty and memory: Toward a unifying theory. Journal of Memory \& Language, 25, 645-656.

McDaniel, M. A., Einstein, G. O., \& Lollis, T. (1988). Qualitative and quantitative considerations in encoding difficulty effects. Memory \& Cognition, 16, 8-14.

Minsky, M. (1975). A framework for representing knowledge. In P. A. Winston (Ed.), The psychology of computer vision (pp. 211-277). New York: McGraw-Hill.

Morris, C. D., Stein, B. S., Bransford, J. D. (1979). Prerequisites for the utilization of knowledge in the recall of prose passages. Journal of Experimental Psychology: Human Leaming and Memory, 5, 253-261.
Naveh-Benjamin, M. (1990). Coding of temporal order information: An automatic process? Journal of Experimental Psychology: Learning, Memory, \& Cognition, 16, 117-126.

NeISSER, U. (1967). Cognitive psychology. New York: Appleton Century-Crofts.

O'BRIEN, E. J., \& MYERS, J. L. (1985). When comprehension difficulty improves memory for text. Journal of Experimental Psychology: Leaming, Memory, \& Cognition, 11, 12-21.

Peterson, R. G., McINTYre, C. W. (1973). The influence of semantic "relatedness" on linguistic information and retention. American Journal of Psychology, 86, 697-706.

RAAijmakers, J. G. W., \& ShIFfrin, R. J. (1981). Search of associative memory. Psychological Review, 85, 59-108.

SChank, R. C., \& Abelson, R. P. (1977). Scripts, plans, goals, and understanding. Hillsdale, NJ: Erlbaum.

Schulman, A. I. (1974). Memory for words recently classified. Memory \& Cognition, 2, 47-52.

Schustack, M. W., ANDERson, J. R. (1979). Effects of analogy to prior knowledge on memory for new information. Journal of Verbal Learning \& Verbal Behavior, 18, 565-583.

SChwarz, M. N. K., \& Flammer, A. (1981). Text structure and titleeffects on comprehension and recall. Joumal of Verbal Learning \& Verbal Behavior, 20, 61-66.

Smith, E. E., AdAms, N., Schorr, D. (1978). Fact retrieval and the paradox of interference. Cognitive Psychology, 10, 438-464.

Stein, B. S., Littlefield, J., Bransford, J. D., Persampieri, M. (1984). Elaboration and knowledge acquisition. Memory \& Cognition, 12, 522-529.

Stein, B. S., Morris, C. D., \& Bransford, J. D. (1978). Constraints on effective elaboration. Journal of Verbal Learning \& Verbal Behavior, 17, 704-714.

Summers, W. V., Horton, D. L., \& Diehl, V. A. (1985). Contextual knowledge during encoding influences sentence recognition. Journal of Experimental Psychology: Learning, Memory, \& Cognition, 11, 771-779.

Tulving, E. (1983). Elements of episodic memory. New York: Oxford University Press.

TzenG, O. J. L., CotTon, B. (1980). A study-phase retrieval model of temporal coding. Journal of Experimental Psychology: Human Learning \& Memory, 6, 705-716.

Tzeng, O. J. L., LeE, A., \& WeTZel, C. D. (1979). Temporal coding in verbal information processing. Journal of Experimental Psychology: Human Learning \& Memory, 5, 52-64.

UNDERWOOD, B. J. (1983). Attributes of memory. Palo Alto: Scott, Foresman.

WALKER, N. (1986). Direct retrieval from elaborated memory traces. Memory \& Cognition, 14, 321-328.

WinOGRAD, E., \& Soloway, R. M. (1985). Reminding as a basis for temporal judgments. Joumal of Experimental Psychology: Learning, Memory, \& Cognition, 11, 262-271.

\section{NOTES}

1. Developed at the Cognitive Laboratory of the University of Oregon by Gil Osgood.

2. This possibility was suggested by an anonymous reader of a previous version of the manuscript.

3. From "Contextual Prerequisites for Understanding: Some Investigations of Comprehension and Recall' by J. D. Bransford and M. K. Johnson, Journal of Verbal Learning \& Verbal Behavior, 1972, 11, 717-726. Copyright 1972 by Academic Press. Reprinted by permission. 


\section{APPENDIX A \\ Stimulus Passages for Experiment 1}

\section{Making and Flyıng a Kite ${ }^{3}$}

A newspaper is better than a magazine. A seashore is a better place than the street. At first it is better to run than to walk. You may have to try several times. It takes some skill but it's easy to learn. Even young children can enjoy it. Once successful, complications are minimal. Birds seldom get too close. Rain, however, soaks in very fast. Too many people doing the same thing can also cause problems. One needs lots of room. If there are no complications, it can be very peaceful. A rock will serve as an anchor If things break loose from it, however, you will not get a second chance.

\section{Starting a Car}

Some adjustments may be necessary. Feet are as important as hands. Restraint is usually recommended. Extremes in temperature may affect the process. Twist in the right direction. A few seconds should be enough. Prolonged attempts that fail are dangerous. Attaching to someone else's may increase your chances. The same symbols should definitely be matched. When the task is complete the red will be gone. Beware if insufficient levels are indicated. Cards can be helpful in overcoming such problems. The proper papers should always be near. Most end exactly where they begin. Everything should be left in the correct position.

\section{APPENDIX B}

\section{Washing Clothes}

The procedure is actually quite simple. First you arrange things into different groups depending on their makeup. Of course, one pile may be sufficient depending on how much there is to do. If you have to go somewhere else due to lack of facilities that is the next step, otherwise you are pretty well set. It is important not to overdo any particular endeavor. That is, it is better to do too few things at once than too many. In the short run this may not seem important, but complications from doing too many can easily arise. A mistake can be expensive as well. The manipulation of the appropriate mechanisms should be self-explanatory, and we need not dwell on it here. At first the whole procedure will seem complicated. Soon, however, it will become just another facet of life. It is difficult to foresee any end to the necessity for this task in the immediate future, but then one never can tell.

Titles of sentences for the individual-sentence-title reading condition:

Sentence Title

Dividing Numbers

Garage Sale

Mixing Concrete

Hotel Reservations

Aerobic Workouts

Loading a Dishwasher

Deep Knee-bends

Building your own House

Playing Video Games

Learning to Drive

Computer Banking

Brushing Teeth
Sentence

The procedure is actually quite simple.

First you arrange things into different groups depending on their makeup. Of course, one pile may be sufficient depending on how much there is to do. If you have to go somewhere else due to lack of facilities that is the next step, otherwise you are pretty well set.

It is important not to overdo any particular endeavor.

It is better to do too few things at once than too many.

In the short run this may not seem important, but complications from doing too many can easily arise.

A mistake can be expensive as well.

The manipulation of the appropriate mechanisms should be self-explanatory, and we need not dwell on it here.

At first the whole procedure will seem complicated.

Soon, it will become just another facet of life.

It is difficult to foresee any end to the necessity for this task in the immediate future, but then one never can tell. 
Stimuli for the composite foil task:

Old

Of course, one pile may be sufficient depending on how much there is to do.

If you have to go somewhere else due to lack of facilities that is the next step, otherwise you are pretty well set.

It is difficult to foresee any end to the necessity for this task in the immediate future, but then one never can tell.

First you arrange things into different groups depending on their makeup.

Soon, it will become just another facet of life.

\section{New}

Of course, one pile may be sufficient, but complications from doing too many things can easily arise.

The manipulation of the appropriate mechanisms should be self-explanatory, otherwise you are pretty well set.

In the short run this may not seem important, but then one never can tell.

First you arrange things into different groups depending on how much there is to do.

A mistake can be just another facet of life.

(Manuscript received October 3, 1990;

revision accepted for publication April 22, 1991.) 